
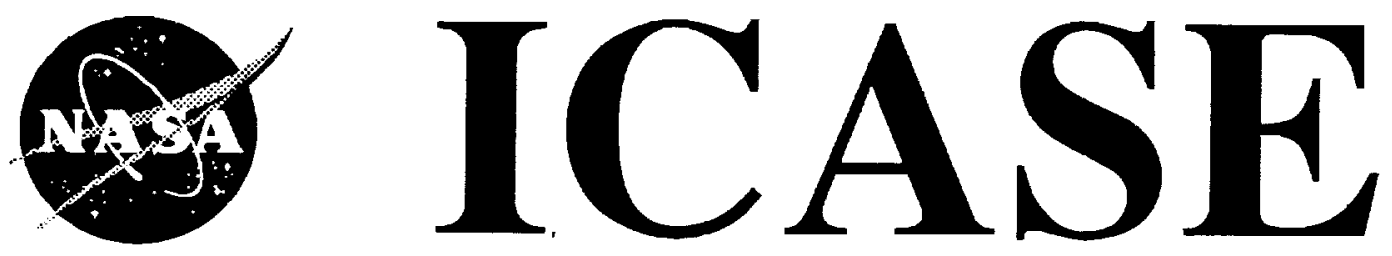

\title{
EFFICIENT BULK-LOADING OF GRIDFILES
}

Scott T. Leutenegger

David M. Nicol

Contract NAS 1-19480

August 1994

Institute for Computer Applications in Science and Engineering NASA Langley Research Center

Hampton, VA 23681-0001

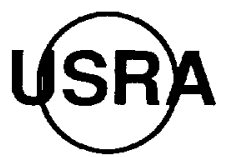

$\begin{array}{lll}a & & \\ \infty & & N \\ \infty & n & 0 \\ m & n & + \\ 1 & 0 & 0 \\ n & u & N \\ a & 5 & 0\end{array}$

$\sum_{0}^{-1}$ 


\title{
Efficient Bulk-Loading of Gridfiles *
}

\author{
Scott T. Leutenegger \\ Institute for Computer Applications in Science and Engineering \\ Mail Stop 132c, NASA Langley Research Center \\ Hampton, VA 23681-0001 \\ leut@icase.edu
}

\author{
David M. Nicol \\ Dept. of Computer Science \\ College of William and Mary \\ Williamsburg, VA 23187-8795
}

\begin{abstract}
$\underline{\text { Abstract }}$
This paper considers the problem of bulk-loading large data sets for for the gridfile multi-attribute indexing technique. We propose a rectilinear partitioning algorithm that heuristically seeks to minimize the size of the gridfile needed to ensure no bucket overflows. Empirical studies on both synthetic data sets and on data sets drawn from computational fluid dynamics applications demonstrate that our algorithm is very efficient, and is able to handle large data sets. In addition, we present an algorithm for bulk-loading data sets too large to fit in main memory. Utilizing a sort of the entire data set it creates a gridfile without incurring any overflows.
\end{abstract}

*This research was supported by the National Aeronautics and Space Administration under NASA contract NAS1-19480 while the authors were in residence at the Institute for Computer Applications in Science and Engineering (ICASE), NASA Langley Research Center, Hampton, VA 23681-0001. Nicol's work was also supported in part by NSF grant CCR-9210372 and NASA grant NAG-1-1132. 


\section{Introduction}

We are developing a scientific database to support retrieval of subsets of Computational Fluid Dynamics (CFD) data sets. Retrieval of subsets is required for visualization and data exploration. All of our data is two or three-dimensional and thus requires multiattribute indexing. We are specifically interested in partially qualified, fully qualified, and point queries. Gridfiles are a well known multi-attribute indexing technique [5]. The basic idea is to partition each attribute range into subranges, thereby inducing a multi-dimensional rectilinear partitioning on the entire multi-attribute space. Enough partitions are chosen to ensure that all tuples sharing the same subrange in each dimension will fit on a disk page. Any point query can be then be satisfied with two disk accesses, one to fetch a pointer to the data page, and one to fetch the data page itself.

The data we wish to store is contained in files created by CFD simulations. Both the size of the data sets and anticipated extensive use of the data sets require that we provide fast organization of new data, and fast retrivial of existing data. Our two dimensional data is typically a large set of tuples of the form:

$$
\left(X, Y, \text { float }_{1}, \text { float }_{2}, \ldots \text { float }_{N}\right)
$$

Current data sets measure only tens of megabytes, but are projected to be $2-3$ orders of magnitude larger soon. Although we are specifically concerned with CFD data sets, large physically oriented data sets are common outputs to a wide spectrum of scientific computations.

In this paper we show how to quickly load entire data files into a gridfile indexing structure. This is termed bulk loading. Note similar functionality is required from relational databases for reloading relations when changing platforms, during recovery, or during reorganization. In a relational database the relation is analogous to the data set in our work.

The main contributions of this paper are:

1. A partitioning algorithm which requires up to two to four orders of magnitude less CPU time than the only known algorithm for partitioning data into gridfile blocks. We provide experimental results for our partitioning algorithm.

2. An efficient algorithm to aggregate under-utilized logical grid-buckets to achieve better disk utilization. We provide expermental results which demonstrate the utility of the aggregation phase.

3. A complete algorithm for bulk-loading of large data sets (significantly larger than main memory) that guarantees no bucket overflows.

The rest of this paper is organized as follows: In the next section we relate our work to prior efforts. In section 3 we present the general problem in more detail and provide an example. In section 4 we present the existing partitioning algorithm, our new algorithm, and our aggregation algorithm. In

section 5 we experimentally compare the execution times of the two algorithms, on a variety of data sets including highly skewed CFD data sets. We also demonstrate the effectiveness of our aggregation technique. In section 6 we present our two phase bulk-loading algorithm. We end with our conclusions and plans for future work. 


\section{Previous Work}

Bulk-loading of $B^{+}$trees [6] has been investigated, but only recently have bulk-loaded grid files been considered. The single paper on this of which we are aware is that of $\mathrm{Li}$, Rotem, and Srivastava [2]. Their main emphasis is bulk-loading of Parallel Grid Files, i.e. grid files that are distributed across multiple sites in a shared nothing environment. They define logical partitioning as that of the gridfile among the sites in the database system, and physical partitioning as that of the portion of a gridfile located at one site, into the buckets that compose that portion of the gridfile. Their solution is based on dynamic programming, for both the logical partitioning and physical partitioning of parallel gridfiles. For physical partitioning their objective function is to minimize bucket overflow. We are concerned only with physical partitioning at a single site, although a modified version of our algorithm could be used for logical partitioning. The $\mathrm{Li}$ et al. algorithm optimally partitions one dimension, given a specific number of partitions and a fixed partitioning in the other dimension (which is likely equally spaced, but details on this fixed partition are lacking the the Li et al. paper). Our algorithm dynamically finds the number of partitions, finds a partitioning much more quickly, and directly addresses the issue of selecting the fixed partition. For uniformly distributed data it may be sufficient to assume an equally spaced partitioning, but this is not the case when data is skewed.

We show that the dynamic programming approach is too inefficient to be considered for large grid files. Li et al. recognize this problem themselves, and suggest sampling $[7,8]$ to accelerate their algorithm. However, sampling may introduce overflows, the handling of which may be significant. For each bucket that overflows an additional bucket must be created and the grid directory split. If the number of overflows within a bucket is larger than the bucket capacity, multiple new buckets will need to be created and the grid directory will be split multiple times. The earlier work inadequately assesses the risks of sampling, focusing as it does on the probability that some block overflows rather than, say, the average number of blocks which overflow and the average total number of overflow tuples.

For the problem specification given in $\mathrm{Li}$ et al. , i.e. given a fixed partitioning and fixed number of partitions, the dynamic programming formulation is an excellent approach, but we propose that it is better to reformulate the problem and find the smallest number of partitions for which the total overflow is zero. The freedom introduced by allowing an arbitrary number of partitions enables us to use a fast heuristic algorithm instead of an expensive dynamic programming algorithm. The possibly larger number of buckets resulting from an increased number of partitions is reduced via a low cost aggregation algorithm. Thus, our partitioning algorithm is capable of handling much larger grid files and still guarantee no overflows while achieving good bucket utilization, although if the data set is too large to fit into main memory the data must first be sorted. Furthermore, we consider more extensive data sets than the earlier work, to better understand the effects of positionally skewed and clustered data which is typical of CFD data sets.

Our partitioning algorithm is a modification of the rectilinear partitioning algorithm developed by Nicol[4] for the purposes of load-balancing irregular data-parallel computations. The two principle differences between our algorithm and this earlier one are that the number of subranges in each dimension are not considered fixed in the present context, and that there is an upper limit on the number of tuples in a bucket. 


\section{General Problem Description}

Before considering algorithmic issues, let us first examine the general problem. Our exposition is of the two-dimensional case; all the algorithms generalize immediately to higher dimensions. We also assume that each attributed range is partitioned into the same number of subranges. This is not rigorously necessary, but we have not addressed how one would choose the desired relationship between number of subranges in each dimension.

Let $S$ be a set of tuples $\left(a_{1}, a_{2}, q[]\right)$ where attributes $a_{1}$ and $a_{2}$ are the indexed attributes and $q[]$ is the rest of the tuple. In our specific data sets $a_{1}$ and $a_{2}$ are $x$ and $y$ coordinates, and $q[]$ is an array of 3-5 floating point values representing physical quantities such as pressure, density, directional derivative information, chemical composition, and so on. For ease of exposition assume the domain of both $a_{1}$ and $a_{2}$ are integers $\in 1, \ldots, n$; the algorithms extend in a straightforward fashion to real-valued attributes and generalized ranges. The empirical results we report are based on these extensions. Let $\mathcal{F}$ be a $n \times n$ frequency matrix which for each entry contains the number of tuples with that coordinate, i. e.

$$
f_{i, j}=\left\|\left\{t \mid t \in S, a_{1}=i, a_{2}=j\right\}\right\|, 1 \leq i, j \leq n
$$

We use the following notation:

$$
\begin{aligned}
& T=\text { the number of tuples in data set } S, \\
& P=\text { the number of partitions in each dimension, } \\
& B=\text { the maximum number of tuples a bucket can hold, } \\
& U_{i}=\text { the number of unique coordinate values in dimension } i, \\
& U_{m a x}=\mathrm{n} i \mathrm{x}\left\{U_{i}\right\}, \\
& C_{i}=\left(c_{i, 1}, c_{i, 2}, \ldots, c_{i, P-1}\right) \text { is the vector of cuts in dimension } i \text {, specifically } C_{1} \text { is the vector of } \\
& \text { horizontal cuts and } C_{2} \text { is the vector of vertical cuts, } \\
& {\left[O_{i, j}\right]=\text { the } P \times P \text { occupancy matrix resulting from applying the cut vectors } C_{1} \text { and } C_{2} \text { to } S,} \\
& \text { total overflow }=\sum_{i=1}^{P} \sum_{j=1}^{P} \max \left\{O_{i, j}-B, 0\right\} .
\end{aligned}
$$

We seek a pair $\left(C_{1}, C_{2}\right)$ whose total overflow equals zero, and whose number of cuts is minimized.

To make these concepts more intuitive, in the left hand side of figure 1 we have the partitioned data set for $S=\{(1,1)(1,3)(1,4)(2,2)(2,8)(3,9)(4,2)(4,3)(5,1)(5,3)(7,2)(7,4)(8,8),(9,3)\}, P=3, C_{1}$ (the horizontal cuts) $=(2,7)$, and $C_{2}=(2,6)$. The partitioning $\left(C_{1}, C_{2}\right)$ divides the domain of $S$ into 9 bins. Note, the dashed lines of $\left(C_{1}, C_{2}\right)$ are slightly offset to clearly show the occupancy of the bins. In this case bin 1 contains points $(1,1)$ and $(2,2)$; bin 2 contains $(1,3)$ and $(1,4)$; bin 3 contains $(2,8)$; bin 4 contains $(4,2),(5,1)$ and $(7,2)$; bin 5 contains $(4,3),(5,3)$, and $(7,4)$; bin 6 contains $(3,9)$; bin 7 is empty; bin 8 contains $(9,3)$; and bin 9 contains $(8,8)$. Thus, the occupancy matrix, $\left[O_{i, j}\right]$, is:

\begin{tabular}{|l|l|l|}
\hline 2 & 2 & 1 \\
\hline 3 & 3 & 1 \\
\hline 0 & 1 & 1 \\
\hline
\end{tabular}



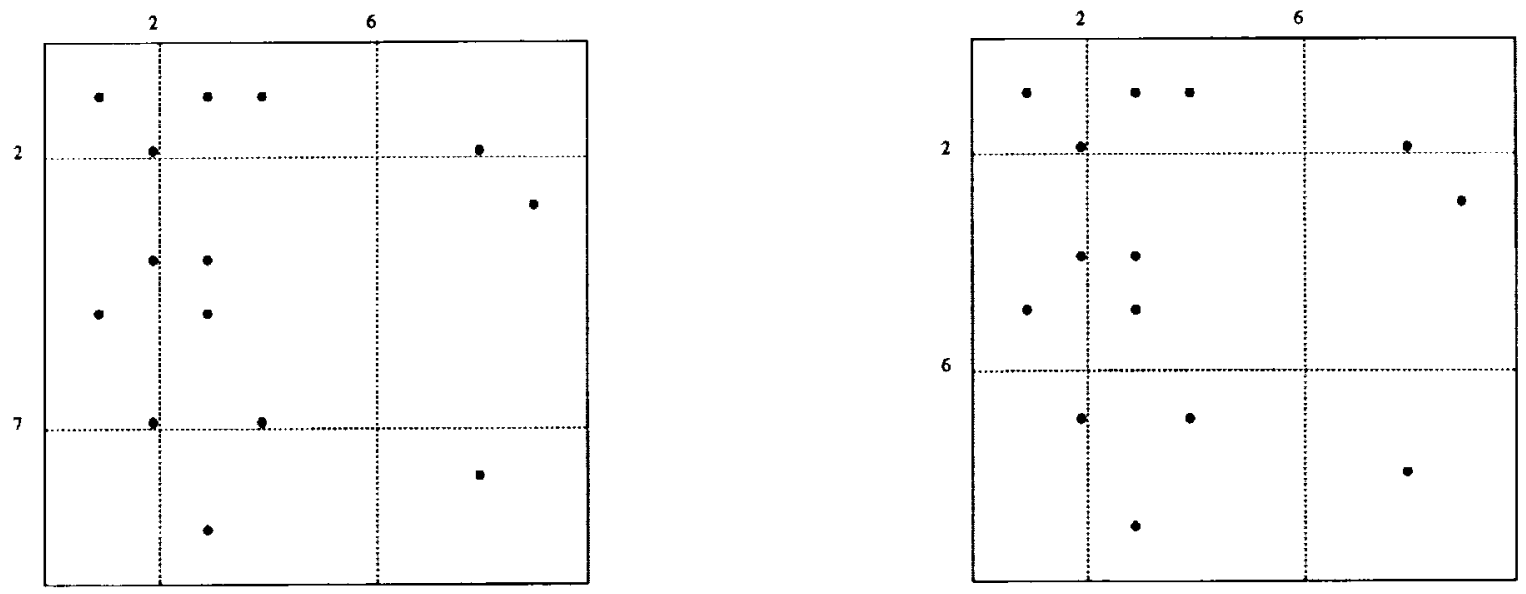

Figure 1: Partitioning Example; Left: total overflow equals 1; Right: total overflow equals 0

If we assume $B=2$, then the total overflow for this partitioning is 2 because bins 4 and 5 each contain 3 points. If we move the position of the second cut of $C_{1}$ to position 6 , i. e. let $C_{1}=(2,6)$, as shown in the right hand side of figure 1 , then the total overflow would be zero.

\section{Algorithm Descriptions}

We now describe the algorithm of Li et. al., and our own. Our implementation of the earlier algorithm is presented in 4.1 in some detail. We provide the detail because it is lacking in the $\mathrm{Li}$ et. al paper, and we wish to show that we've made every effort to optimize the performance of their dynamic programming solution. Section 4.2 gives our own partitioning algorithm, while 4.3 describes our method for aggregating under-utilized buckets.

\subsection{Dynamic Programming Solution}

The dynamic programming equation to be described is precisely the one given in Li et al. [2]. We reword that formulation and describe specifics of an optimized algorithm for solving that equation.

It is assumed that $S$ is already partitioned in the horizontal dimension, i.e. $C_{1}$ is fixed. Our task is to find a vector $C_{2}$ that minimizes the total overflow. Let $\mathcal{R}(i, j)$ be the $n \times(j-i+1)$ submatrix of $\mathcal{F}$ obtained by restricting $a_{2}, i \leq a_{2} \leq j$. Now consider the column of bins resulting from partitioning $\mathcal{R}(i, j)$ horizontally by $C_{1}$. Let $O V_{1}(i, j)$ be the sum, over each member of this column, of the bin overflow. For example, with $B=2$ and the matrix on the left of figure $1, O V_{1}(2,3)$ equals 2 since the middle bin has 4 tuples, and no overflow is observed in the other two bins. To reduce overflows we might consider partitioning $\mathcal{R}(i, j)$ vertically with $l-1$ cuts, thereby creating a $P \times l$ submatrix of bins with an attendant total overflow value. There may be many ways of partitioning columns $i$ through $j$ of $\mathcal{R}(i, j)$ with $l-1$ cuts; let $T O V_{1}(i, j, l)$ be the minimum possible total overflow cost among all these 
possibilities. The principle of optimality [1] then asserts that

$$
\operatorname{TOV}_{1}(1, j, l)= \begin{cases}\operatorname{Min}_{i=l-1, \ldots, j-1}\left\{\operatorname{TOV}_{1}(1, i, l-1)+O V_{1}(i+1, j)\right\}, & 1<l \leq j, \\ O V_{1}(1, j), \quad l=1 & \end{cases}
$$

Of particular interest is the value $T O V_{1}(1, T, P)$, and the partition that achieves this cost.

Solution of this equation is aided by precomputing values from which each $O V_{1}(i, j)$ can be derived in $O(P)$ time, as follows. $C_{1}$ partitions $\mathcal{F}$ into $P$ submatrices, $S_{1}, \cdots, S_{P}$. For each $S_{k}$ and column index $j$ define $r_{k}(1, j)$ to be the sum of entries in $S_{i}$ between column indices 1 and $j$, inclusive. Then, for any pair of column indices $i$ and $j$ we have $r_{k}(i, j)=r_{k}(1, j)-r_{k}(1, i-1)$. Now

$$
O V_{1}(i, j)=\sum_{k=1}^{P} \max \left\{r_{k}(i, j)-B, 0\right\} .
$$

Since $r_{k}(i, j)$ is computed with a single subtraction, $O V_{1}(i, j)$ is computed in $O(P)$ time. The set of all $r_{k}(1, j)$ values can be computed in time proportional to $n \log (P)$. With only slightly more computation (a sort in each dimension) we can accommodate tuple sets that are sparse relative to $n \times n$. We project the data set onto each coordinate axis and sort it, essentially working with a $T \times T$ array containing only $T$ non-zeros. The indices we describe in this paper may be thought of as the ordinal positions of the data projections on each axis. We take advantage of the sparse structure and still compute all $r_{k}(1, j)$ values time proportional to $T \log (P)$.

The dynamic programming equation expresses a recursion in both column index $j$, and number of cuts, $l$. Our approach is to unravel the recursion with $j$ being the inner index, and $l$ the outer one. Specifically, we start by solving $T O V_{1}(1, j, 1)$ for all $j$; given these we solve $T O V_{1}(1, j, 2)$ for all $j$, and so on. For $l>1$ when solving $\operatorname{TOV}_{1}(1, j, l)$ we must make up to $j-l$ comparisons (actually, we must make one compari, n tor every non-zero column of $\mathcal{F}$ between columns $l-1$ and $j-1$ ). If the tuple sets are not sparse relative to $n \times n$, the complexity of the inner loop of the recursion is $O\left(P n^{2}\right)$, and the outer loop is executed giving a complexity of $O\left(P^{2} n^{2}\right)$. In addition, the complexity of the initial precalculation of the $r_{k}(1, j)$ is $O(T \log (P))$, thus the total complexity is $O\left(P^{2} n^{2}+T \log (P)\right)$. If the data sets are sparse relative to $n \times n$, then the complexity can be reduced to $O\left(P^{2} U_{2}^{2}+U_{2} \log (P)+T \log (T)\right)$, where $U_{2}$ is the number of unique attribute values in dimension 2, and the additional $T \log (T)$ is for sorting the tuples which is needed to maintain the sparse representation. In the rest of this paper we will assume the data sets are sparse relative to $n \times n$. Sparse data sets are especially relevant since the coordinates of our unstructured CFD data sets are reals. The asymptotic complexity is $O\left(\max \left\{P^{2} U_{2}^{2}, T \log T\right\}\right)$. We will henceforth call this algorithm the DP algorithm.

The speed of the algorithm can be further increased by precalculating and storing all the values $O V_{1}(i, j) \forall i, \forall j$. The complexity is then $O\left(P U_{2}^{2}+U_{2} \log (P)+T \log (T)\right)$. The precalculation of the $O V_{1}(i, j)$ requires time proportional to $O\left(P U_{2}^{2}\right)$, and is thus included in that term. This storage cost can be very significant and hence limits the applicability of this optimization. For example, if $U_{2}$ is 5000 , the space required for storing the $O V_{1}(i, j)$ is 95 megabytes. We will henceforth call this algorithm the DP2 algorithm.

We have now described how to calculate the optimal overflow cost and partitioning of $S$ given fixed partitioning $C_{1}$. So far the only difference from our work and that of Li et al. is that we have provided 
the details of our implementation of the dynamic programming problem. We now come to the first contribution of this paper, how to determine the fixed partitionings and how to determine the number of partitions.

We assume that the number of partitions in each dimension is the same, thus resulting in square gridfile directories. We presume the existence of an algorithm which, given a fixed set of cuts in one dimension finds a "good" set of cuts in the other dimension. The paper by Li et al. provides one such, but neglects to specify the origin of the fixed cut set. We follow Nicol [4] by using such an algorithm as the basis for an iterative method: Given fixed cuts in one dimension, find good cuts in the other. Treat the new cuts as fixed, and find better ones in the previously fixed dimension. The iterations are maintained until some termination mechanism triggers. The initial fixed cut is uniformly spaced. In the gridfile application of this idea, each application of the cut-finding algorithm attempts to find cuts that yield zero overflow at all buckets. Termination of such a partitioning session is defined when either an overflow-free cut-set is discovered, or after some specified number of iterations (we use 20) no such cut-set is discovered. The sole parameter to a partitioning session is the number of partitions, $P$, in each dimension. A partitioning session may be viewed as a probe that determines whether we can quickly discovered an overflow-free partitioning using $P-1$ cuts in each dimension. Our overall strategy is to do an intelligent search on $P$ to find the smallest value for which we can quickly determine a desirable partitioning.

Any cut assignment might be used in the approach above. The results we later report use both the dynamic programming solution of $\mathrm{Li}$ et al., and our own algorithm (to be reported) within this same framework. For skewed data sets it may be advantageous to have the number of partitions in each dimension differ, but our aggregation phase described later minimizes the poor efficiency of using square regions. In the future we intend to investigate non-square regions. Given a square region, strict lower and upper bounds on the number of partitions needed in each dimension are:

$$
\begin{gathered}
\text { lowerBound }=(\lfloor T / B\rfloor)^{0.5} \\
\text { upperBound }=T
\end{gathered}
$$

We thus can do a binary search to find the minimal number of partitions $P$, lower Bound $\leq P \leq$ upper Bound, for which the total overflow is equal to zero. In practice, we have found it is faster start with the number of partitions equal to $2 \times$ lowerBound. Then, while the total overflow is greater than zero keep doubling the number of partitions. Once a partition value has been found for which the total overflow is zero, conduct a binary search with that value as the upper bound, and the previous value as the lower bound.

\subsection{Rectilinear Partitioning}

We now come to the second contribution of our work, an alternative rectilinear partitioning algorithm. Like that of Li et al., it optimizes the cuts in one dimension given a fixed set of cuts in the other. In the discussion to follow we take $C_{1}$ as fixed.

At each step of the algorithm we seek to define a column of buckets whose width is as wide as possible without any bucket in the column being assigned more than $B$ tuples. To define the first column 
we seek the largest index $j$ for which $O V_{1}(1, j)=0$; call this index $j_{1}$. Since $O V_{1}(1, j)$ is monotone non-decreasing in $j$, we may identify $j_{1}$ with a binary search. Using the precalculated $r_{k}(i, j)$, each candidate $j$ requires $O(P)$ time to compute $O V_{1}(1, j)$, hence $O\left(P \log U_{2}\right)$ time is required to define the

first column. The second column is computed exactly as the first, only taking index $j_{1}+1$ as the starting point, i.e., identify the largest $j_{2}$ for which $O V_{1}\left(j_{1}+1, j_{2}\right)=0$. This process continues until either $P$ or fewer adjacent overflow-free columns are discovered, or all $P-1$ cuts are placed and the last column suffers overflow. In the former case the partitioning session terminates; in the latter case we may freeze the newly discovered cuts and choose new cuts in the other dimension. The complexity of one partitioning session has several components. First there is an $O(T \log T)$ cost for sorting the tuples in each dimension. Now for each time we optimize in one dimension we first compute new $r_{k}(1, j)$ values, which takes $O(T \log (P))$ time. This is followed by a $O\left(P^{2} \log U_{2}\right)$ cost for allocating cuts. Since any partitioning session iterates a bounded number of times, the asymptotic complexity is $O\left(\max \left\{P^{2} \log U_{2}, T \log T\right\}\right)$.

The original rectilinear application [4] was shown to converge to unchanging cut sets (given sufficiently many iterations). Our algorithm too would converge, but we have found it more prudent to back away to a larger number of partitions when a small number of iterations fails to find a suitable partition. The original rectilinear partitioning problem was shown to be NP-hard in three dimensions; the same proof suffices to show the intractability of finding minimal $P$ for which a square overflow-free partition exists. The tractability of the rectilinear partitioning problem in two dimensions is still unknown.

It is informative to consider an essential difference between our partitioning algorithm and that of $\mathrm{Li}$ et al. We are uninterested in any partition that has overflow, and so expend no computational energy on minimizing non-zero overflows. If, given $C_{1}$ it is possible to find $C_{2}$ yielding an overflow-free partition, our algorithm will find it. If none exists, our algorithm determines that quickly. By contrast, the previous algorithm seeks to find $C_{2}$ that minimizes overflow. We are uninterested in whether the minimal overflow is two or three, only whether it is zero or non-zero. This distinction permits us to find overflow-free partitions with substantially less work than the previous algorithm, as will be seen in the empirical results.

\subsection{Aggregation}

Our third contribution is an algorithm for aggregating adjacent buckets with low utilization. After the partitioning phase some of the buckets may have low utilization. If two adjacent buckets both have $50 \%$ utilization or smaller we may combine them into a single bucket (even though the gridfile directory will contain two pointers-they will be identical). Following partitioning, we apply an aggregation scheme based on this observation.

Let $B$ equal the bucket capacity. First assume the grid directory is of size $2^{i} \times 2^{i}$, and view it as four equal sized $2^{i-1} \times 2^{i-1}$ quadrants labeled NW,NE,SE,SW. Define a procedure CanMerge $(A, B, j)$ that returns logical true if neither $A$ nor $B$ has already been merged into some group at level $j$ and their sum of utilization is less than $100 \%$. Define procedure Merge $(A, B, j)$ to merge $A$ and $B$ into one bucket at level $j$. Using CanMerge and Merge we define a recursive function function $\operatorname{Aggregate}(A, j)$ as follows. 


\begin{tabular}{|c|c|c|c|}
\hline 1 & 2 & 8 & 7 \\
\hline 1 & 2 & 3 & 6 \\
\hline 0 & 1 & 4 & 5 \\
\hline 2 & 0 & 2 & 7 \\
\hline
\end{tabular}

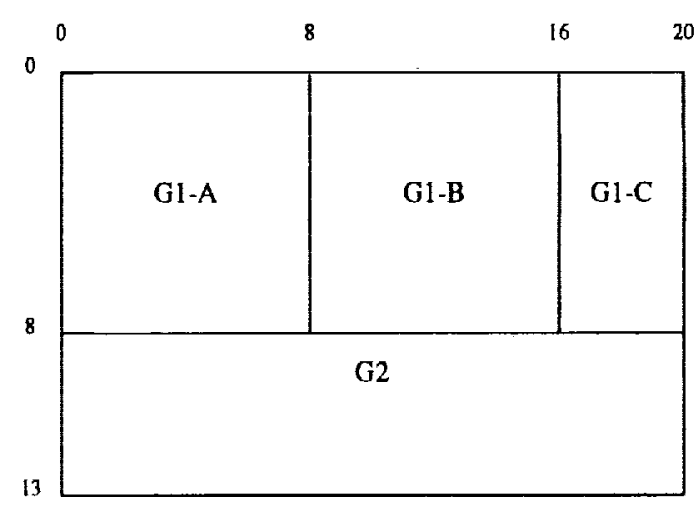

Figure 2: Aggregation Examples

1. If $A$ consists of a $1 \times 1$ gridfile or if $A$ has already been merged into some group at level $j-1$, return.

2. Partition $A$ into four quadrants, NW,NE,SE,SW.

3. If the sum of utilizations of all four quadrants is less than $100 \%$, aggregate them all into one bucket, return.

4. if CanMerge(NW,NE,j) AND CanMerge(SW,SE, $\mathrm{j})$ then: call Merge $(N W, N E, j)$, Merge(SW,SE,j)

5. if CanMerge $(N W, S W, j)$ AND CanMerge(NE,SE,j) then: call Merge $(N W, S W, j)$, Merge(NE,SE,j)

6. if CanMerge $(N W, N E, j)$ then: call Merge $(N W, N E, j)$

7. if CanMerge(SW,SE, j) then: call Merge(SW,SE, $)$

8. if CanMerge $(N W, S W, j)$ then: call Merge $(N W, S W, j)$

9. if CanMerge(NE,SE,j) then: call Merge(NE,SE,j)

10. call Aggregate $(N W, j+1), \quad$ Aggregate $(N E, j+1), \quad$ Aggregate $(S W, j+1), \quad$ Aggregate $(S E, j+1)$

Assuming the grid file directory $D$ is initially $2^{i} \times 2^{i}$, the aggregation is accomplished with the call Aggregate $(D, i)$.

As an example consider the grid directory in the left hand side of figure 2 and a bucket capacity of 10. Entries in the directory are the number of tuples in the bucket. We can not merge the whole into one bucket, nor can we merge as two halves, but we can merge the NW and SW quadrants and then call the aggregation strategy on the two remaining quadrants.

In practice there is no restriction to powers of two. Although our current partitioning algorithm assumes the grid has an equal number of partitions in each dimension we present our aggregation 
algorithm in the most general case. Without loss of generality assume the shape of the grid directory is $N$ rows by $M$ columns, where $N<M$. We find the largest $i$ such that $2^{i}<N$. Let G1 be the $2^{i} \times M$ subdirectory of the grid directory composed of the first $2^{i}$ rows, and let G2 be the $N-2^{i} \times M$ subdirectory composed of the complement of the original directory. We first aggregate G1. Let $\mathrm{j}=$ $M \operatorname{div} N$, this is the number of square $2^{i} \times 2^{i}$ subdirectories that can fit in $\mathrm{G} 1$. For each one of these $\mathrm{j}$ square subdirectories we apply the square region aggregation algorithm above. We are then left with a $2^{i} \times\left(M-j \cdot 2^{i}\right)$ subdirectory and G2. We apply the algorithm recursively on these two regions. In the right hand side of figure 2 we show an example for a $13 \times 20$ grid directory. Subdirectory G1 is composed of G1-A, G1-B, and G1-C. The square power of two region aggregation policy above is applied to G1-A and G1-B, while the entire aggregation policy is called recursively on G1-C and G2. This algorithm could be improved to yield slightly better bucket utilizations, but is very fast and has proved to sufficient for our needs so far.

Depending on the use of the gridfile, different aggregation strategies can be used. If the gridfile is read only, as in our CFD database, then the buddy-system pairing approach needed to facilitate splits for future insertion of tuples is not necessary. In this case regions of aggregated buckets need not be rectangular and hence could allow for more aggregation resulting in improved bucket utilization. We have not yet developed any algorithms to calculate this aggregation since the above algorithm has been sufficient for our needs to date. On the other hand, if the gridfile is being used in a transaction processing environment and tuples might later be inserted, the buddy pairing must be preserved.

\section{Experimental Comparison}

In this section we present experimental results for the two partitioning algorithms. We present both run times and bucket utilization results. In all of our experiments we do not make any attempt to get smooth curves or collect confidence intervals. The figures are the result of one experimental run and thus often have some noise, presumably from use of the workstation by other jobs. All experiments were run on a Sparc 10 workstation.

Sanity checks on the code were made by running both algorithms through a profiler to make sure time was being spent in sections of the code where expected. The run time of the RP algorithm is dominated by the startup costs of creating the pre-calculated $r_{k}(1, j)$ and sorting the records. For most of the data sets in this paper over $40 \%$ of the run time is spent creating the $r_{k}(1, j)$ and over $20 \%$ of the time sorting the data points. Note that even with this high cost of creating $r_{k}(1, j)$, the overall algorithm significantly faster than when the $r_{k}(1, j)$ are not precalculated. In contrast, the run time of the DP algorithm is dominated by the actually partitioning since it is $O\left(P^{2} U_{\text {max }}^{2}\right)$.

In section 5.1 we present results for a single partitioning given a fixed partitioning in the other dimension. In the following sections we present results assuming the number of partions and the initial partitioning is not known. In section 5.2 we present results when the from uniformly distributed synthetic data sets, while in section 5.3 we present results for highly skewed CFD data sets. In section 5.4 we present the bucket utilization results from our experiments and demonstrate the utility of the aggregation phase. 


\subsection{Fixed Partitioning Given}

We first compare the DP, DP2, and RP algorithms assuming that a fixed partitioning exists in one dimension. We conduct these experiments since this is the exact scenario for which Li et al. proposed their algorithm. Note again that how this fixed partitioning is obtained is not specified in Li et al. [2].

We consider a data set of 5,000 tuples where the $\mathrm{x}$ and $\mathrm{y}$ coordinates of each tuple are each chosen from a uniform distribution from 1 to 2000. We obtain the initial horizontal partitioning by equally spacing the cuts within the domain. In table 1 we present results for the number of partitions in each dimension varied from 12 to 5 assuming a bucket capacity of 50 tuples. The columns headed "seconds" record the amount of CPU time used for the partitioning, columns headed "overflow" are the total number of tuples that did not fit within the bucket capacity, and the columns headed "BlocksOver" are the number of blocks which overflowed. The overflow and BlocksOver numbers are identical for the DP and DP2 algorithms since the algorithms find the exact same partitioning and only differ in run time. First note that the RP algorithm is one to two orders of magnitude faster than the DP and DP2 algorithms for all values of $\mathrm{P}$. Conversely, the dynamic programming algorithms minimize total overflow better when there is a large number of partitions. Thus, for the specific problem and objective function as formulated by $\mathrm{Li}$ et al. the dynamic programming algorithm proposed satisfies the objective function better than our rectilinear partitioning algorithm, but at the expense of significantly more computation. A premise of our work is that it is better to partition with a sufficiently large number of partitions to ensure no overflows.

Note that although the DP algorithm does have a smaller number of tuples overflowed, it results in a larger number of buckets which overflow when the number of partitions is less than 11. The blocks which overflow when the RP algorithm is used are all in the last column of the partitioning, whereas when the DP algorithm is used the overflow blocks are spread out in the partitioning space. Consider the case where the number of partitions is 10 . When the RP algorithm is used there are 10 overflow blocks. These 10 blocks have 106, 106, 101, 111, 94, 94, 108, 112, 113, and 106 tuples allocated to them. Since only 50 tuples fit per block 18 new blocks will need to be created. One the other hand, when the DP algorithm is used there are 40 overflow blocks, each of which has at most 68 tuples, requiring 40 new blocks to be created. Hence, total overflow is not a good indicator of the optimality of a partitioning. We propose that a better metric would be the number of new blocks needed to hold the overflows. We will continue to use total tuple overflow in this paper since our algorithms dynamically find the number of partitions needed to make the overflow zero.

\subsection{Number of Partitions Not Given: Uniformly Distributed Data}

We now assume that the number of partitions is not known and that no initial fixed partitioning is given. We first consider the run time of the algorithm for uniformly distributed data. The $x$ and $y$ coordinates of each tuple are each chosen from a uniform distribution from 1 to $\mathrm{N}$, where $\mathrm{N}$ depends on the experiment. In all reported experiments we do not allow any duplicate data set points since our CFD data does not have any duplicate points. We have verified that inclusion of duplicates results in similar relative performance. We first consider the relative performance of the algorithms as the number of tuples is varied. 


\begin{tabular}{|c|ccc|cccc|}
\hline & \multicolumn{3}{|c|}{ RP Algorithm } & DP & DP2 & & \\
P & seconds & overflow & BlocksOver & seconds & seconds & overflow & BlocksOver \\
\hline 12 & $6.50 \mathrm{e}-01$ & 0 & 0 & $2.47 \mathrm{e}+02$ & $1.36 \mathrm{e}+02$ & 0 & 0 \\
11 & $6.30 \mathrm{e}-01$ & 98 & 9 & $2.19 \mathrm{e}+02$ & $1.27 \mathrm{e}+02$ & 14 & 5 \\
10 & $6.70 \mathrm{e}-01$ & 1051 & 10 & $1.80 \mathrm{e}+02$ & $1.23 \mathrm{e}+02$ & 239 & 40 \\
9 & $6.20 \mathrm{e}-01$ & 1672 & 9 & $1.59 \mathrm{e}+02$ & $1.13 \mathrm{e}+02$ & 950 & 76 \\
8 & $6.50 \mathrm{e}-01$ & 2321 & 8 & $1.97 \mathrm{e}+02$ & $9.87 \mathrm{e}+01$ & 1800 & 56 \\
7 & $6.30 \mathrm{e}-01$ & 2958 & 7 & $1.22 \mathrm{e}+02$ & $8.94 \mathrm{e}+01$ & 2550 & 43 \\
6 & $6.20 \mathrm{e}-01$ & 3465 & 6 & $9.54 \mathrm{e}+01$ & $8.02 \mathrm{e}+01$ & 3200 & 31 \\
5 & $6.80 \mathrm{e}-01$ & 3940 & 5 & $7.43 \mathrm{e}+01$ & $7.07 \mathrm{e}+01$ & 3750 & 20 \\
\hline
\end{tabular}

Table 1: CPU Times and Overflow, Fixed Partitioning Given

In figures 3 and 4 we plot the computation time in seconds versus the number of tuples in the relation assuming coordinate values are uniformly distributed from 1 to 2000 . Note that the $y$-axis is logarithmic. From top to bottom we plot the computation time of the DP, DP2, and RP algorithms. Remember that the DP2 algorithm is the same as the DP algorithm except it precomputes and stores the $O V_{1}(i, j) \forall i \forall j$. The plot in figure 3 assumes 50 tuples fit per page, the plot in figure 4 assumes 300 tuples per page. If page size is 8192 bytes then tuples size would be 164 and 27 bytes respectively. A tuple size of 164 bytes may be a typical size for transaction processing systems, and tuples in our data sets are usually around 24-32 bytes. As the number of tuples increases the run time of the DP algorithm becomes too long to be of practical use. A relation of 40,000164 byte tuples is only 6.4 mega-bytes, for 32 byte tuples this is only 1.2 mega-bytes, hence it is reasonable to expect there to be sufficient memory to partition data sets of at least 40,000 tuples.

For 40,000 164 byte tuples, figure 3, the DP algorithm requires 26600 seconds (about 7.4 hours), and 100,000 tuples require 77200 seconds (21.4 hours). These times are clearly prohibitive. The DP2 algorithm requires 3000 seconds (50 minutes) and 6070 seconds (101 minutes) for 40,000 and 100,000 tuples respectively, but it requires 15 mega-bytes of space to hold the precomputed $O V_{1}(i, j)$. The RP algorithm only requires 12 and 40 seconds for 40,000 and 100,000 tuples respectively. Thus, the RP algorithm is a practical algorithm. The RP algorithm is about 2000 (250) times faster than the DP (DP2) algorithm for 40,000 tuples. The difference in solution times is not unexpected given the complexities of the DP, DP2, and RP algorithms which are $O\left(\max \left\{P^{2} U_{2}^{2}, T \log T\right\}\right), O\left(\max \left\{P U_{2}^{2}, T \log T\right\}\right)$, and $O\left(\max \left\{P^{2} \log U_{2}, T \log T\right\}\right)$ respectively.

We now consider how the number of unique attribute values in the data set impacts the relative performance of the policies. In figure 5 we plot the computation time in seconds versus the maximum of the attribute domain for a data set with 40,000 tuples and assuming 300 tuples fit per page. Note that the y-axis is logarithmic. The curves from top to bottom are for the DP, DP2, and RP algorithms. We did not run the DP2 algorithm when the storage space for the precalculated $O V(i, j)$ exceeded 80 mega-bytes, thus there are no points plotted for maximum domain values of 5,000 and higher. Increasing the maximum domain value increases the number of unique attribute values in the data set. The DP and DP2 algorithms are highly sensitive to the number of unique values in the data set. Conversely, the RP algorithm is relatively insensitive to the number of unique values. When the maximum domain value is 2,000, the RP algorithm is 450 (110) times faster than the DP (DP2) algorithm. When the 
maximum domain value is 10,000 , the $\mathrm{RP}$ algorithm is 17,000 times faster than the DP algorithm. All other experiments in this section assume a maximum domain value of 2000 . For many of our CFD data sets the number of unique values is almost equal to the number of tuples, thus even 10,000 is a very small value.

We now consider how the tuple size effects the relative performance of the two algorithms. In figure 6 we plot the computation time in seconds versus the number of tuples per page assuming 40,000 tuples with an attribute domain maximum of 2000 . Once again the y-axis is logarithmic. As the number of tuples per page decreases, hence the tuple size increases, the DP algorithms requires significantly more computation. Conversely, the RP algorithm is relatively insensitive to the size of the tuples. Thus, the RP algorithm remains a viable algorithm for a wide range of tuple sizes. The degradation of the DP algorithm as tuple size increases is easy to predict from the complexity of the algorithm: $O\left(P^{2}\left(U_{\max }\right)^{2}+\left(U_{\max }\right) \log (P)\right)$. As tuple size increases the number of tuples per bucket decreases and hence the number of partitions, $P$, increases. We would expect the runtime of the RP algorithm to increase also since the complexity of the RP algorithm is $O\left(P^{2} \log \left(U_{m a x}\right)\right)$, but the majority of the run time of the RP algorithm is spent sorting the tuples and creating the $r_{k}(1, j)$, thus obscuring the sensitivity to tuple size.

In figure 7 we plot the ratios of the computation time of the DP and DP2 algorithms relative to the RP algorithm. As the tuple size increases the ratio increases.

\subsection{Number of Partitions Not Given: Unstructured CFD Data}

We now consider the run time of the algorithm for highly skewed data. We use actual data sets from unstructured grid CFD simulations. Here the term grid is used to describe the way the coordinates in the data set are connected. The data set is composed of $x, y$ real-valued coordinates. The data sets are from computational models of cross sections of airflows around aircraft wings [3]. In figure 8 we plot the data set for a set with 1034 points where $x \in(-10 \ldots 10), y \in(-12 \ldots 12)$, and restrict the range plotted since the majority of the data is in the central region and plotting the whole range would make it difficult to distinguish the points in areas of high concentration. Only 94 of the 1034 points are not plotted. The vertical and horizontal lines are the partitioning lines resulting from running the RP algorithm on the data set. Note, there is one vertical line at $\mathrm{x}=6.09$ which is not included in the plot. As can be seen from the partitioning, a fixed equal space partitioning would be a bad choice.

In figure 9 we plot the partitioning computation time versus the number of tuples for three different data sets. For the smallest data set, 1034 tuples, the DP (DP2) algorithm required 2370 (650) times more computation than the RP algorithm for partitioning. For the data set with 3959 tuples, the DP (DP2) algorithm required 38,817 (5629) times more computation than the RP algorithm. Thus, the DP algorithm is especially impractical for highly skewed data. Since the DP algorithm required 42 hours for the 3959 tuples data set we did not run the 15895 tuple data set. The RP algorithm required 66 seconds to partition a 15,895 tuple data set.

The four orders of magnitude difference in computation time is not surprising in light of the results from the experiment plotted in figure 5. For unstructure grid data sets the number of unique attribute values is almost equal to the number of tuples, hence as the number of tuples in the set increases not 


\begin{tabular}{|c|c|c|c|c|c|c|}
\hline \multirow[b]{3}{*}{$\mathbf{n}$} & \multicolumn{3}{|c|}{ RP Algorithm } & \multicolumn{3}{|c|}{ DP Algor thm } \\
\hline & & Bucket & tilization & & Buclet & tilization \\
\hline & Partitions & pre-aggregation & post-aggregation & Partitions & pre-aggregation & post-aggregation \\
\hline$\overline{1000}$ & 5 & $8.00 \mathrm{e}-01$ & $8.70 \mathrm{e}-01$ & 5 & $8.00 \mathrm{e}-01$ & $8.00 \mathrm{e}-01$ \\
\hline 5000 & 12 & $6.94 \mathrm{e}-01$ & $7.94 \mathrm{e}-01$ & 12 & $6.94 \mathrm{e}-01$ & $7.81 \mathrm{e}-01$ \\
\hline 10000 & 16 & $7.81 \mathrm{e}-01$ & $7.84 \mathrm{e}-01$ & 16 & $7.81 \mathrm{e}-01$ & $8.06 \mathrm{e}-01$ \\
\hline 20000 & 24 & $6.94 \mathrm{e}-01$ & $7.43 \mathrm{e}-01$ & 23 & $7.56 \mathrm{e}-01$ & $7.60 \mathrm{e}-01$ \\
\hline 40000 & 33 & $7.35 \mathrm{e}-01$ & $7.47 \mathrm{e}-01$ & 33 & $7.35 \mathrm{e}-01$ & $7.45 \mathrm{e}-01$ \\
\hline 60000 & 41 & $7.14 \mathrm{e}-01$ & $7.27 \mathrm{e}-01$ & 40 & $7.50 \mathrm{e}-01$ & $7.54 \mathrm{e}-01$ \\
\hline 80000 & 48 & $6.94 \mathrm{e}-01$ & $7.31 \mathrm{e}-01$ & 47 & $7.24 \mathrm{e}-01$ & $7.37 \mathrm{e}-01$ \\
\hline 100000 & $\overline{53}$ & $7.12 \mathrm{e}-01$ & $7.27 \mathrm{e}-01$ & 52 & $7.40 \mathrm{e}-01$ & $7.43 \mathrm{e}-01$ \\
\hline
\end{tabular}

Table 2: Average Bucket Utilizations, Number of Tuples Varied

only does the number of partitions needed increase, but so does the number of unique attribute values. The RP algorithm does not experience as much of an increase in computation time as the data sets get larger since the majority of its time is spent in the precalculation of the $r_{k}(1, j)$ and the initial sort of the data.

\subsection{Bucket Utilizations and Aggregation Effectiveness}

We now present the average bucket utilizations for some of the previous experiments both before and after our aggregation phase is completed. In table 2 we present the utilizations for the uniformly distributed data experiment in figure 3. The column label "Partitions" is the number of partitions in each direction. This was the smallest number for which the algorithm returned a total overflow of zero. Overall the average bucket utilization is quite good, about the same as would result from inserting the tuples one at a time. There is little difference between the utilization for the DP and RP algorithms. In addition, the aggregation phase does not significantly improve the bucket utilization. This is because the bucket utilization is already good. For most experiments, the run time of the aggregation phase is minimal, less than $2 \%$ of the RP runtime, hence it is worth aggregating even for a modest improvement.

In table 3 we present the utilizations for the uniformly distributed data experiment in figure 6 . Once again there is little difference in bucket utilization for the two algorithms. The average bucket utilization tends to decrease as the number of tuples per page decreases. When only 5 tuples fit per page the bucket utilization is only $28 \%$, but after the aggregation it is better than $70 \%$. Thus, the aggregation phase can considerably improve the utilization for cases where the utilization is poor. The runtime of the DP algorithm for 5 and 10 tuples per page was excessive and hence we do not present aggregation results for those parameters.

For skewed data the aggregation phase results in substantial savings of disk space. In table 4 we present the utilizations for the unstructured grid CFD data set for three different grids. The average bucket utilization without aggregation is very poor but improves significantly with aggregation. Thus, for highly skewed data aggregation is essential for achieving good bucket utilizations. Note, there is no 15,896 tuple data for the DP algorithm since its computation time on the 3959 tuple data set required 42 hours. 


\begin{tabular}{|c|c|cc||c|cc|}
\hline & \multicolumn{3}{|c||}{ RP Algorithm } & \multicolumn{3}{c|}{ DP Algorithm } \\
\hline $\begin{array}{c}\text { Tuples } \\
\text { per-page }\end{array}$ & Partitions & \multicolumn{2}{|c|}{$\begin{array}{c}\text { Bucket Utilization } \\
\text { pre-aggregation }\end{array}$} & post-aggregation & Partitions & \multicolumn{2}{c|}{ Bucket Utilization } \\
pre-aggregation & post-aggregation \\
\hline \hline 300 & 13 & $7.89 \mathrm{e}-01$ & $8.33 \mathrm{e}-01$ & 12 & $9.26 \mathrm{e}-01$ & $9.26 \mathrm{e}-01$ \\
\hline 200 & 15 & $8.89 \mathrm{e}-01$ & $8.89 \mathrm{e}-01$ & 15 & $8.89 \mathrm{e}-01$ & $8.89 \mathrm{e}-01$ \\
\hline 100 & 22 & $8.26 \mathrm{e}-01$ & $8.26 \mathrm{e}-01$ & 22 & $8.26 \mathrm{e}-01$ & $8.26 \mathrm{e}-01$ \\
\hline 50 & 33 & $7.35 \mathrm{e}-01$ & $7.47 \mathrm{e}-01$ & 33 & $7.35 \mathrm{e}-01$ & $7.45 \mathrm{e}-01$ \\
\hline 25 & 51 & $6.15 \mathrm{e}-01$ & $6.93 \mathrm{e}-01$ & 49 & $6.66 \mathrm{e}-01$ & $7.14 \mathrm{e}-01$ \\
\hline 10 & 94 & $4.53 \mathrm{e}-01$ & $7.10 \mathrm{e}-01$ & & & \\
\hline 5 & 169 & $2.80 \mathrm{e}-01$ & $7.05 \mathrm{e}-01$ & & & \\
\hline
\end{tabular}

Table 3: Average Bucket Utilizations, Tuples Per Page Varied

\begin{tabular}{|c|c|cc||c|cc|}
\hline & \multicolumn{3}{|c||}{ RP Algorithm } & \multicolumn{2}{c|}{ DP Algorithm } \\
\cline { 2 - 6 }$t$ & Partitions & \multicolumn{2}{|c|}{ Bucket Utilization } & & \multicolumn{2}{c|}{ Bucket Utilization } \\
pre-aggregation & post-aggregation & Partitions & pre-aggregation & post-aggregation \\
\hline \hline 1034 & 10 & $2.07 \mathrm{e}-01$ & $6.27 \mathrm{e}-01$ & 10 & $2.07 \mathrm{e}-01$ & $7.95 \mathrm{e}-01$ \\
\hline 3959 & 34 & $6.85-02$ & $7.61-01$ & 29 & $9.41 \mathrm{e}-02$ & $5.87 \mathrm{e}-01$ \\
\hline 15896 & 131 & $1.85 \mathrm{e}-02$ & $5.76 \mathrm{e}-01$ & & & \\
\hline
\end{tabular}

Table 4: Average Bucket Utilizations, Unstructured Grid CFD Data

\section{Two-Phase Bulk Loading Algorithm Description}

In this section we describe a two phase algorithm for bulk loading of data sets significantly larger than available buffer space. Suppose the data set contains $S$ tuples, and suppose that a maximum of $A$ tuples can be contained in memory at a time when applying the RP algorithm. Our approach has two steps. First we partition the set into groups of size $A$ or fewer. Each set will contain all points within a rectangle in the $\mathrm{x}-\mathrm{y}$ plane; however the collection of sets need not be rectilinear. In the second step we apply RP to each individual set, and merge the individual grid files created. These steps are now elaborated upon.

Given $S$ and $A$ we find the smallest perfect square integer $R$ such that $R>\frac{S}{A}$. We will partition the data set into $R$ groups, as follows. By sorting the data set on the $\mathrm{x}$-coordinate value we may easily divide the set into $\sqrt{R}$ groups of $\sqrt{R}$ successive elements in the sorted order. This serves to partition the data set along the x-axis into "strips" of tuples. Each such strip may be sorted along the y-axis, after which its points may be separated into groups of successive $\frac{S}{A}$ points. This effectual divides a strip into rectangles, with no rectangle containing more than the permitted number of points.

It remains to apply RP to each group, and write the buckets of data to disk. One possibility is to partition each group separately, and define the final grid file as the union of all separately defined gridfiles. Recognizing that a cut which is defined for a group on one side of the data domain must propagate through $\sqrt{R}$-1 other groups (and cause splitting of grid directories in each) we consider a different approach. As the groups are partitioned we build up a global grid file, initially empty. Upon reading in a group we identify the set of cuts in the global grid file which affect this group, treat them as immutable, and seek to find the minimum number of additional cuts needed to avoid overflow. This requires a simple modification to the $\mathrm{RP}$ algorithm. 
Another optimization is to first strip the attributes being indexed from the data set. Then the two phase algorithm is applied to the coordinates without requiring $\mathrm{I} / \mathrm{O}$ of the whole tuple. After partitioning the set of coordinates and creating the overall grid directory, the buckets could be filled by making a second pass over the data set. This may result in a faster load time if the tuple size is large.

If the data set (and hence the grid directory) is extremely large, another optimization uses a two level directory scheme as suggested in [5] where the top level directory has one entry for each of the $R$ sub-directories. Note, this would mean that a point access could require three disk accesses instead of two.

\section{Conclusions and Future Work}

We have proposed and implemented a new rectilinear partitioning (RP) algorithm for physical partitioning of gridfiles. Our proposed RP algorithm is significantly faster than the recently proposed dynamic partitioning (DP) algorithm of $\mathrm{Li}$ et al. [2]. The number of overflows $\mathrm{RP}$ permits is necessarily larger than the DP algorithm (which minimizes them), however we argue that minimizing the number of additional blocks created due to overflow is actually a better measure, and is one for which the RP algorithm finds better solutions that the DP algorithm.

We considered the use of our greedy algorithm and the DP algorithm as kernels in a loop that seeks to minimize the size of the grid file needed to achieve no overflows. For synthetic data sets of uniformly distributed integers the RP algorithm is two to three orders of magnitude faster than the DP algorithm. For actual CFD data sets, whose indexed attributes are highly skewed reals, the RP-based algorithm is three to four orders of magnitude faster than the DP-based algorithm.

We have also developed an efficient aggregation algorithm for improving bucket utilizations of gridfiles resulting from bulk loading using the RP or DP partitioning algorithms. The algorithm has minimal overhead, and can yield substantial improvements in bucket utilization when the bucket utilization after partitioning is poor. This aggregation phase is necessary to achieve reasonable bucket utilizations when the indexed data is highly skewed.

We have also proposed a two phase bulk load algorithm and several optimizations for loading data sets that are significantly larger then the available buffer space. This algorithm guarantees no bucket overflows and is proposed as a possible alternative to sampling based methods. We have yet not investigated the performance of the algorithm.

In the future we plan to experimentally compare our two phase algorithm with inserting one tuple at a time and sampling based methods. We also intend to consider more sophisticated aggregation techniques and partitioning with differing numbers of partitions for each attribute.

\section{References}

[1] T.H. Horowitz, S. Sahni, Fundamentals of Computer Algorithms, Computer Science Press, 1978. 
[2] Li, J., Rotem, D., Srivastave, J., "Algorithms for Loading Parallel Grid Files," Proceedings of ACMI SIGMIOD 1993, p. 347-356, Washington D.C., 1993.

[3] D..J. Mavriplis, "Algebraic Turbulence Modeling for Unstructured and Adaptive Meshes," American Institute of Aeronautics and Astronautics (AIAA) Journal vol. 29, no. 12, p. 2086-2093, December 1991.

[4] Nicol, D.M., "Rectilinear Partitioning of Irregular Data Parallel Computations," ICASE Report 91-55, NASA Contractor Report \#187601, July 1991, to appear in the Journal of Parallel and Distributed Computation.

[5] Nievergelt, J., Hinterberger, H., Sevcik, K.C., "The Grid File: An Adaptable, Symetric Multikey File Structure," ACM Transactions on Database Systems, vol. 9, no. 1, March 1984, p. 38-71.

[6] Rosenberg, A.L., Snycler, L., "Time and Space Optimality in B-Trees," ACM Trasactions on Datbase Systems, vol. 6, no. 1, March 1981.

[7] Seshadri, S., "Probalistic Method in Query Processing,", Ph. D. thesis, Department of Computer Science, University of Wisconsin-Madison, 1992.

[8] Seshadri, S., Naughton, J.F., "Sampling Issues in Parallel Datal,ase Systems," Proceeding of the 3rd International Conf. on Extending Database Technology, Vienna, Austria, March 1992. 


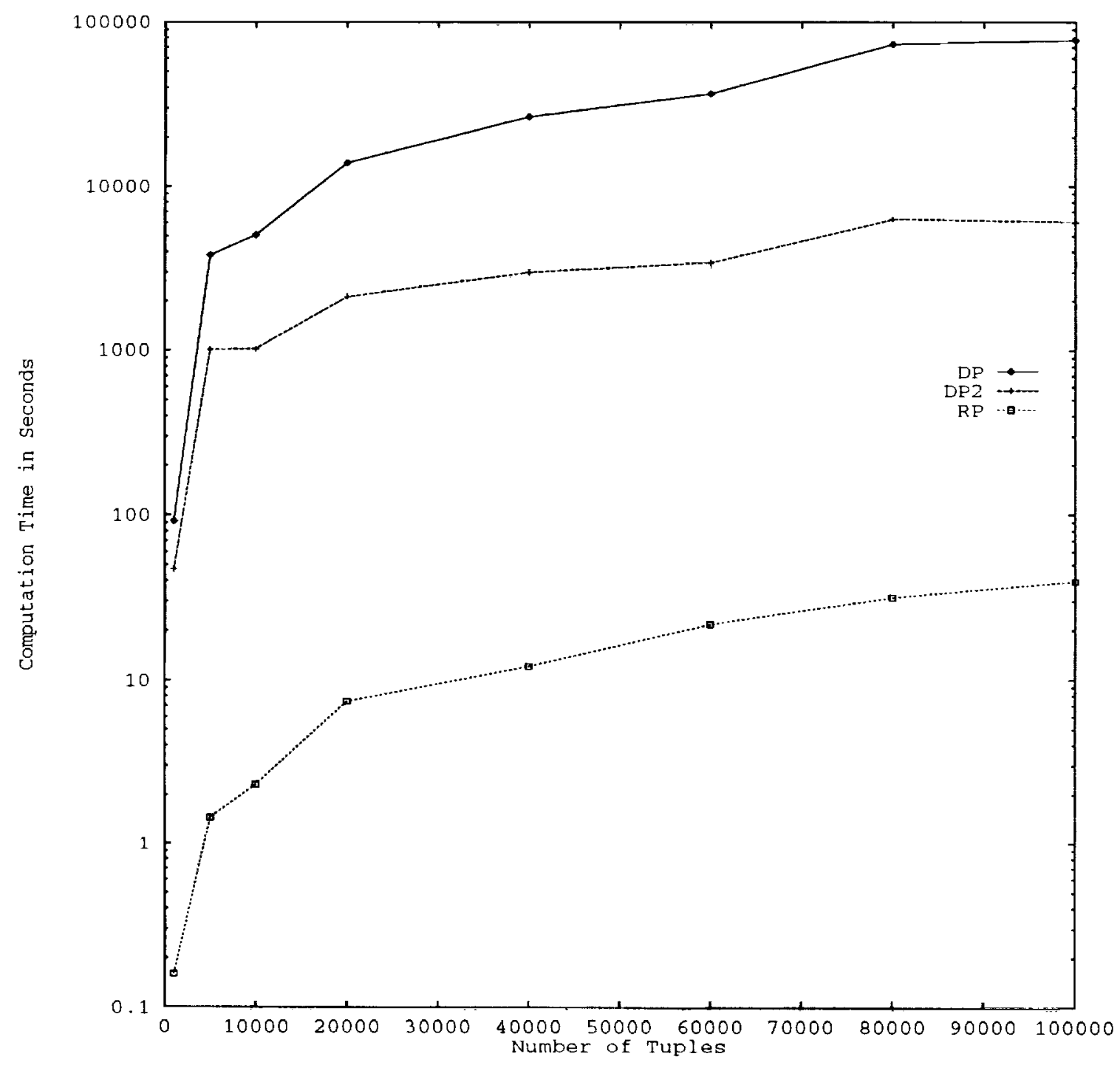

Figure 3: Number of Tuples Varied, 50 tuples per page, Domain Maximum $=2000$ 


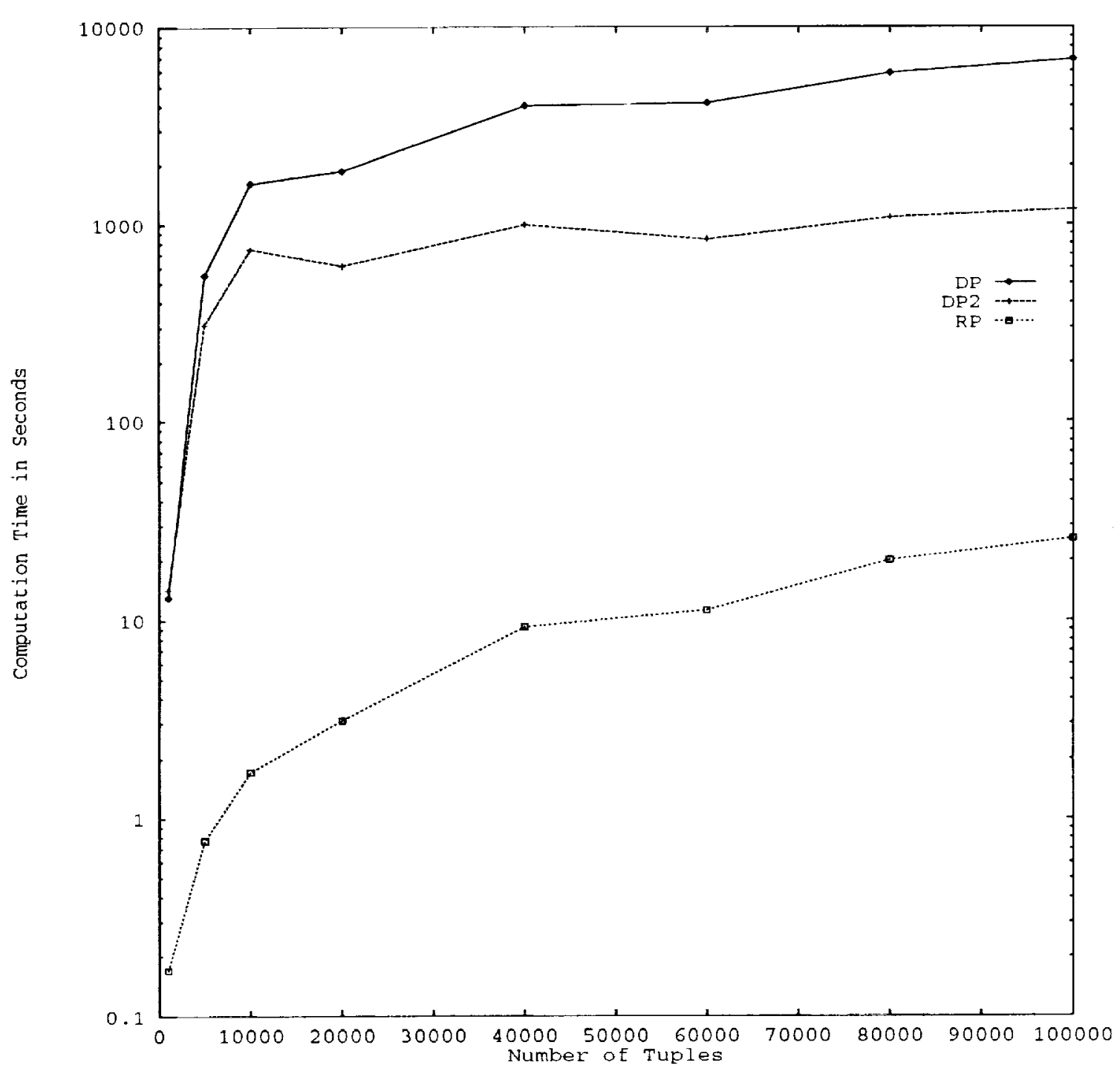

Figure 4: Number of Tuples Varied, 300 tuples per page, Domain Maximum $=2000$ 


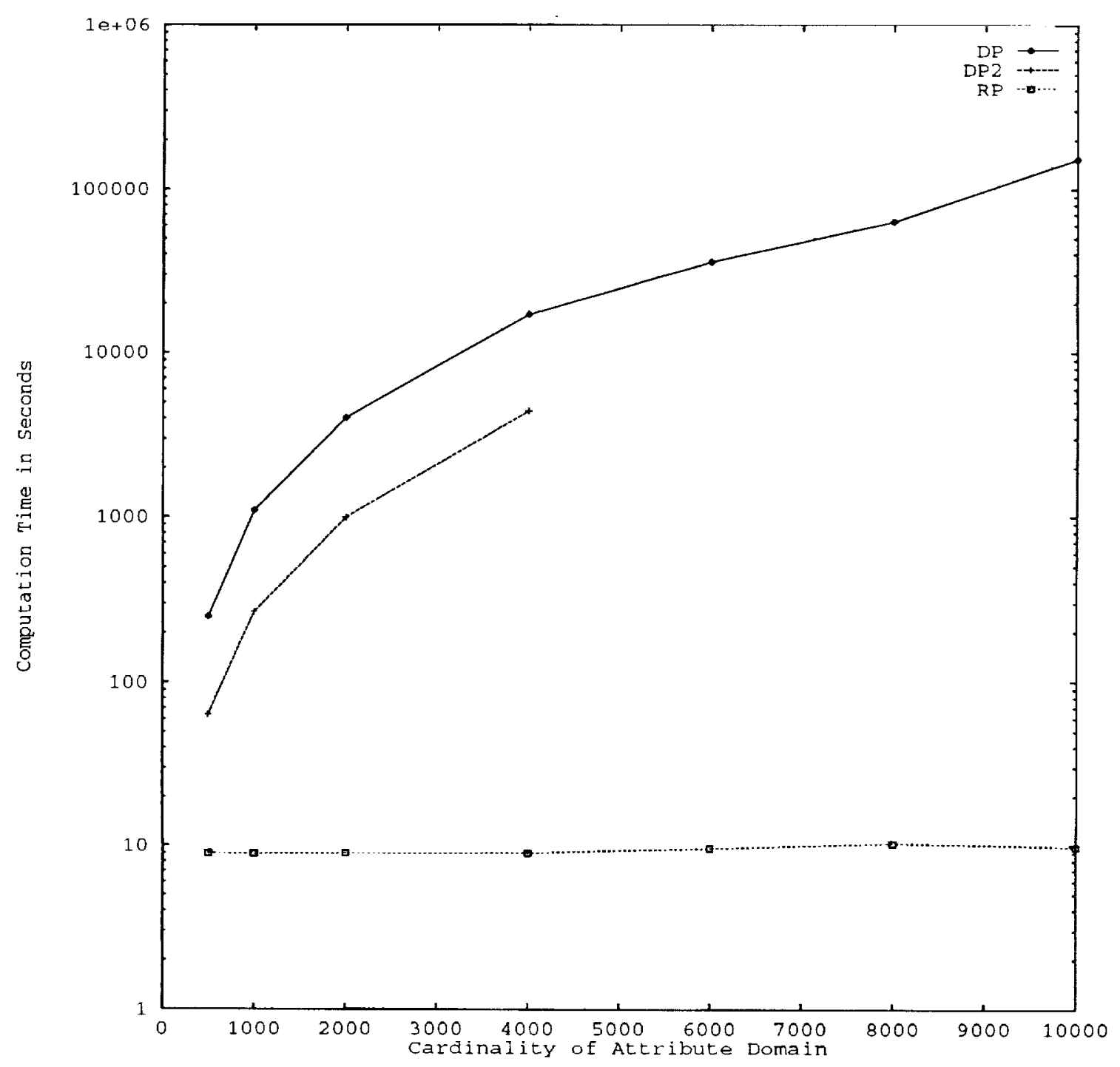

Figure 5: Maximum of Attribute Domain Varied; 40,000 Tuples, 300 tuples per page 


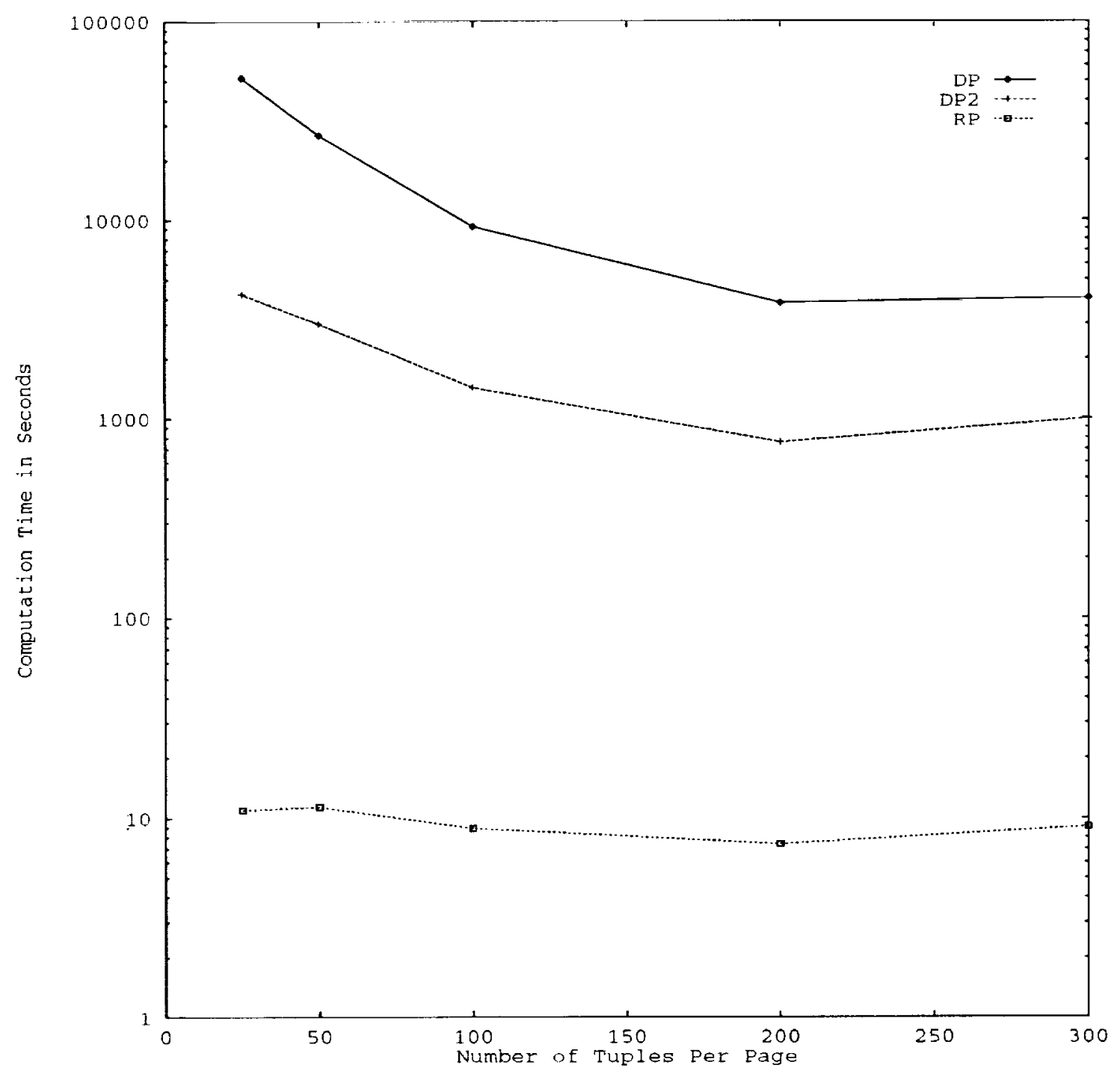

Figure 6: Size of Tuples Varied; 40,000 Tuples, Range of attribute $=1 . .2000$ 


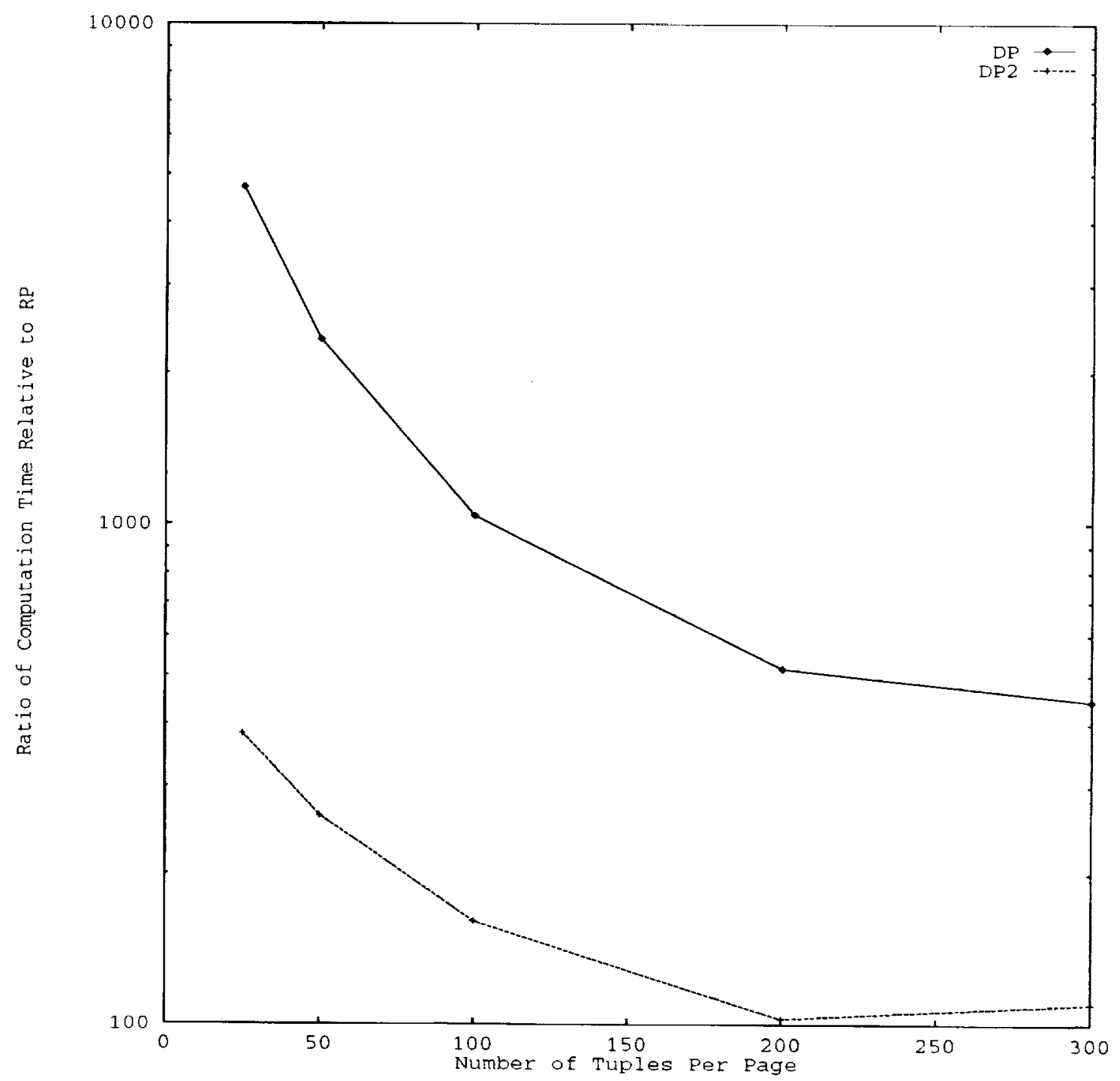

Figure 7: Size of Tuples Varied; 40,000 Tuples, Range of attribute $=1$.. 2000 


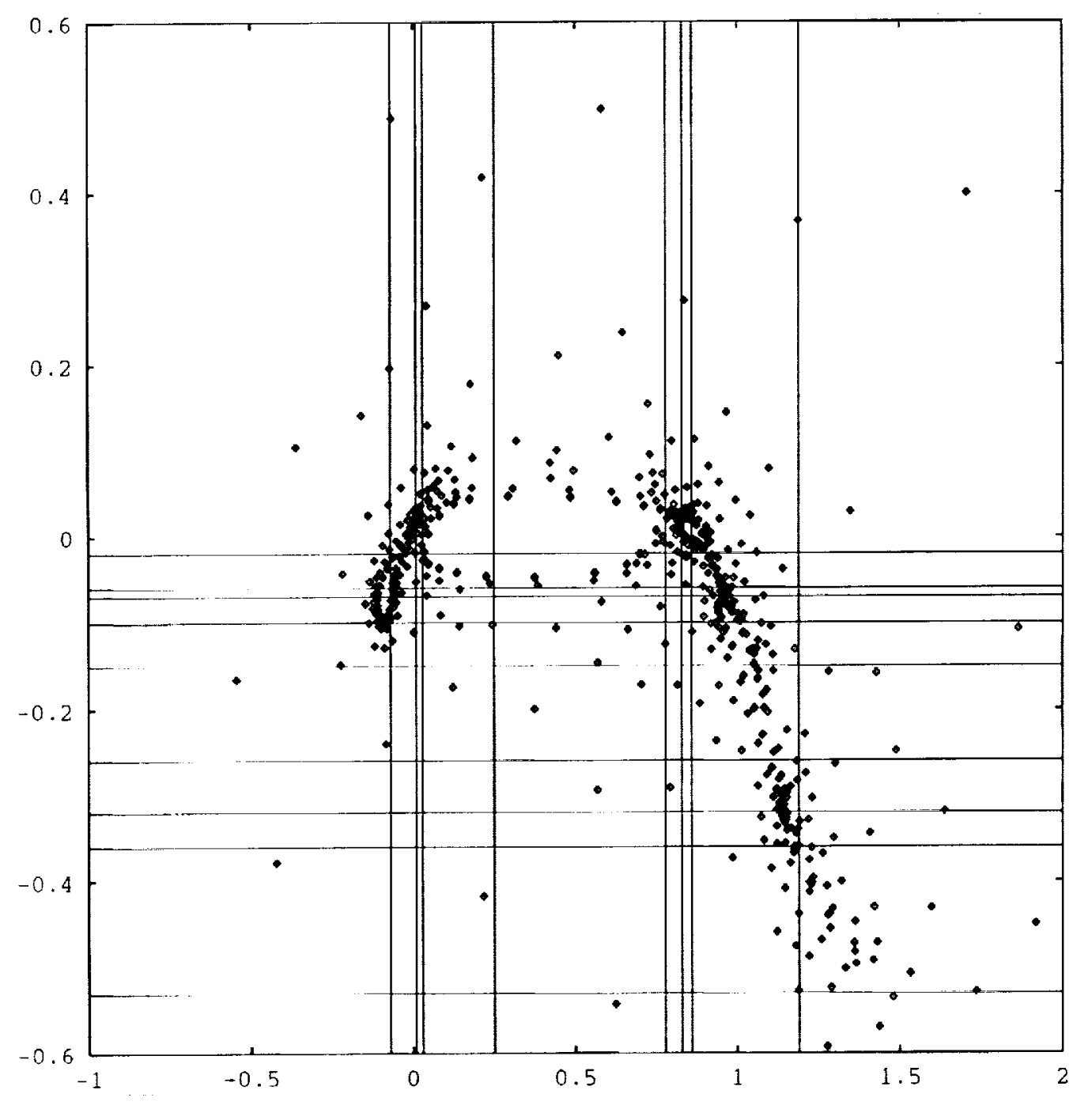

Figure 8: Plot of Unstrucuted Grid CFD Data Set 


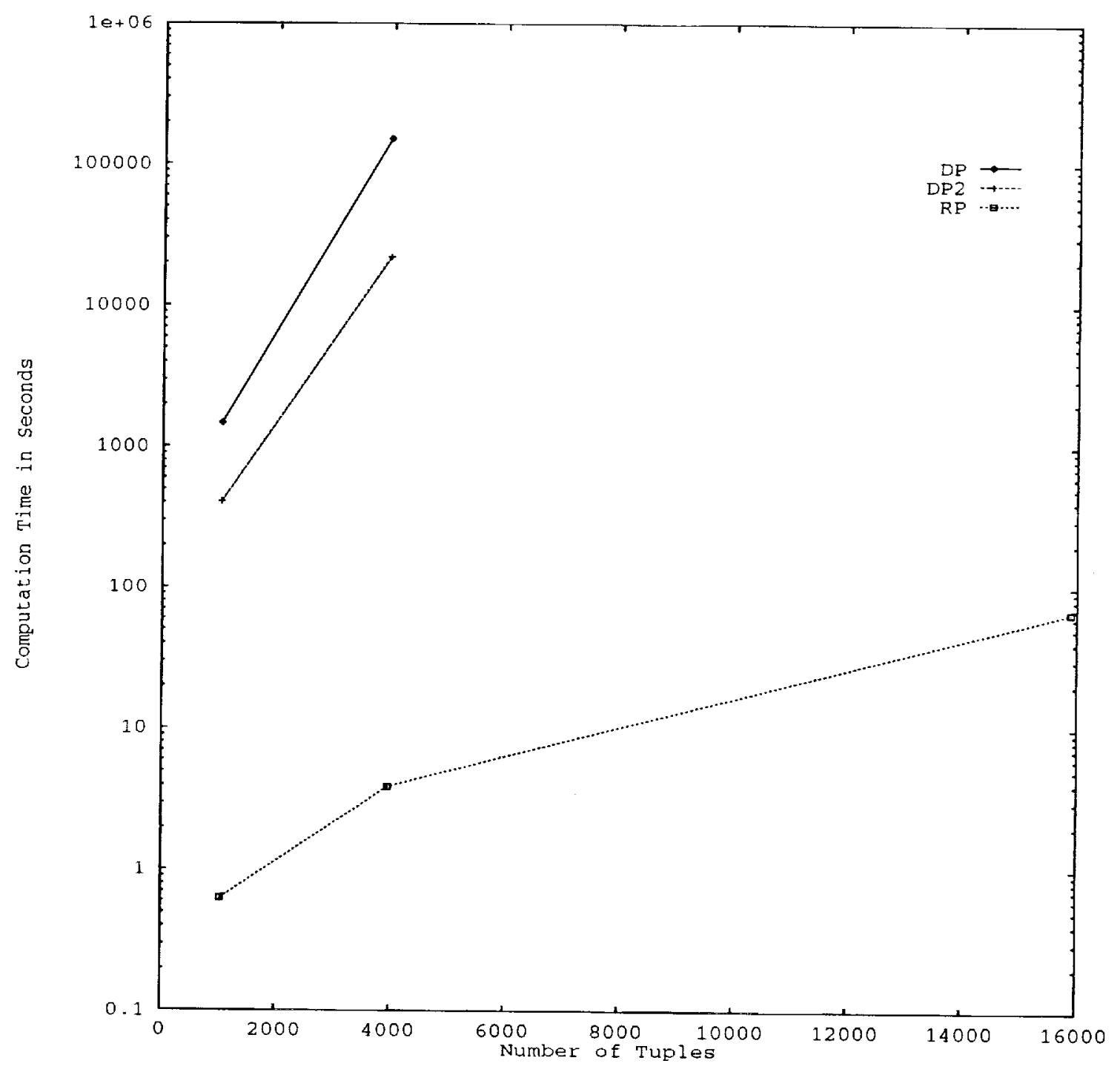

Figure 9: Partitioning time for Unstrucuted Grid CFD Data Sets 


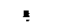




\begin{tabular}{|c|c|c|c|}
\hline \multicolumn{3}{|c|}{ REPORT DOCUMENTATION PAGE } & $\begin{array}{l}\text { Form Approved } \\
\text { OMB No. 0704-0188 }\end{array}$ \\
\hline \multicolumn{4}{|c|}{ 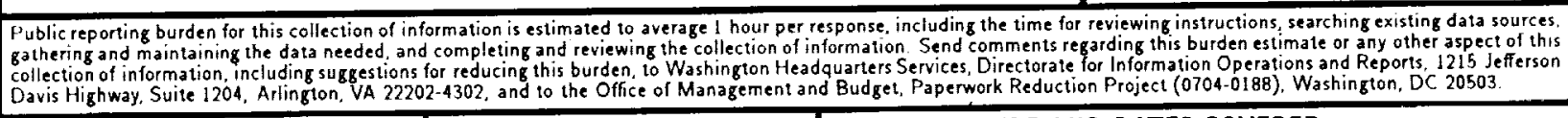 } \\
\hline 1. AGENCY USE ONLY(Leave blank) & $\begin{array}{l}\text { 2. REPORT DATE } \\
\text { August } 1994\end{array}$ & \multicolumn{2}{|c|}{$\begin{array}{l}\text { 3. REPORT TYPE AND DATES COVERED } \\
\text { Contractor Report }\end{array}$} \\
\hline \multicolumn{3}{|c|}{$\begin{array}{l}\text { 4. TITLE AND SUBTITLE } \\
\text { EFFICIENT BULK-LOADING: OF GRIDFILES }\end{array}$} & \multirow[t]{2}{*}{$\begin{array}{l}\text { 5. FUNDING NUMBERS } \\
\text { C: NAS1-19480 } \\
\text { WU } 505-90-52-01\end{array}$} \\
\hline \multicolumn{3}{|l|}{$\begin{array}{l}\text { 6. AUTHOR(S) } \\
\text { Scott T. Leutenegger } \\
\text { David M. Nicol }\end{array}$} & \\
\hline \multicolumn{3}{|c|}{$\begin{array}{l}\text { 7. PERFORMING ORGANIZATION NAME(S) AND ADDRESS(ES) } \\
\text { Institute for Computer Applications in Science } \\
\text { and Engineering } \\
\text { Mail Stop } 132 \text { C, NASA Langley Research Center } \\
\text { Hampton, VA 23681-0001 }\end{array}$} & $\begin{array}{l}\text { 8. PERFORMING ORGANIZATION } \\
\text { REPORT NUMBER } \\
\text { TCASE Report No. } 94-74\end{array}$ \\
\hline \multicolumn{3}{|c|}{$\begin{array}{l}\text { 9. SPONSORING/MONITORING AGENCY NAME(5) ANO ADDRESS(ES) } \\
\text { National Aeronautics and Space Administration } \\
\text { Langley Research Center } \\
\text { Hampton, VA } 23681-0001\end{array}$} & $\begin{array}{l}\text { 10. SPONSORING/MONITORING } \\
\text { AGENCY REPORT NUMBER } \\
\text { NASA C'R-194974 } \\
\text { ICASE Report No. } 94-74\end{array}$ \\
\hline \multicolumn{4}{|c|}{$\begin{array}{l}\text { 11. SUPPLEMENTARY NOTES } \\
\text { Langley Technical Monitor: Michael F. Card } \\
\text { Final Report } \\
\text { Submitted to IEEE Transaction on Knowledge and Data Engineering }\end{array}$} \\
\hline \multicolumn{3}{|c|}{$\begin{array}{l}\text { 12a. DISTRIBUTION/AVAILABILITY STATEMENT } \\
\text { Unclassified-Unlimited } \\
\text { Subject Category } 60,61\end{array}$} & 12b. DISTRIBUTION CODE \\
\hline \multicolumn{4}{|c|}{$\begin{array}{l}\text { 13. ABSTRACT (Maximum } 200 \text { words) } \\
\text { This paper considers the problem of bulk-loading large data sets for the gridfile multi-attribute indexing technique. } \\
\text { We propose a rectilinear partitioning algorithm that heuristically seeks to minimize the size of the gridfile needed to } \\
\text { ensure no bucket overflows. Empirical studies on both synthetic data sets and on data sets drawn from computational } \\
\text { fluid dynamics applications demonstrate that our algorithm is very efficient, and is able to handle large data sets. } \\
\text { In addition, we present an algorithm for bulk-loading data sets too large to fit in main memory. Utilizing a sort of } \\
\text { the entire data set it creates a gridfile without incurring any overflows. }\end{array}$} \\
\hline \multirow{2}{*}{\multicolumn{2}{|c|}{$\begin{array}{l}\text { 14. SUBJECT TERMS } \\
\text { ('iridfile, bulk-loading, Scientific Databases }\end{array}$}} & 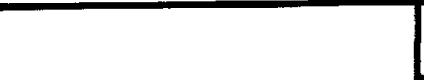 & $\begin{array}{l}\text { 15. NUMBER OF PAGES } \\
25\end{array}$ \\
\hline & & & $\begin{array}{r}\text { 16. PRICE CODE } \\
\mathrm{A} 03 \\
\end{array}$ \\
\hline $\begin{array}{l}\text { 17. SECURITY CLASSIFICATION } \\
\text { OF REPORT } \\
\text { Unclassified }\end{array}$ & $\begin{array}{l}\text { 18. SECURITY CLASSIFICATION } \\
\text { OF THIS PAGE } \\
\text { Tnclassified }\end{array}$ & $\begin{array}{l}\text { 19. SECURITY CLASSIFICATION } \\
\text { OF ABSTRACT }\end{array}$ & $\begin{array}{l}\text { 20. LIMITATION } \\
\text { OF ABSTRACT }\end{array}$ \\
\hline NSN $7540-01-280-5500$ & & & $\begin{array}{l}\text { Standard Form } 298(\text { Rev. } 2-89) \\
\text { Prescribed by ANSI Std. Z39-18 } \\
\text { 298-102 }\end{array}$ \\
\hline
\end{tabular}

